TRANSACTIONS OF THE

AMERICAN MATHEMATICAL SOCIETY

Volume 353, Number 2, Pages 479-489

S 0002-9947(00)02636-

Article electronically published on October 11, 2000

\title{
CODING INTO $K$ BY REASONABLE FORCING
}

\author{
RALF-DIETER SCHINDLER
}

\begin{abstract}
We present a technique for coding sets "into $K$," where $K$ is the core model below a strong cardinal. Specifically, we show that if there is no inner model with a strong cardinal then any $X \subset \omega_{1}$ can be made $\boldsymbol{\Delta}_{3}^{1}$ (in the codes) in a reasonable and stationary preserving set generic extension.
\end{abstract}

\section{INTRODUCTION}

The present paper was inspired by a talk Itay Neeman gave on his joint work 5] and 6] with J. Zapletal. Starting from a large cardinal dose somewhat above $A D^{L(\mathbb{R})}$ (the assertion that $L(\mathbb{R})$ is a model of the Axiom of Determinacy) they show that no set of ordinals not already in $L(\mathbb{R})$ can be coded into $L(\mathbb{R})$ by a setsized reasonable forcing, and moreover that the theory of $L(\mathbb{R})$ with parameters for ordinals and reals from $V$ is frozen with respect to all generic extensions by set-sized reasonable forcings. "Reasonability" was introduced by Foreman and Magidor in [2]. A notion of forcing $P$ is called reasonable if for any $\alpha \geq \omega_{1}$ it is true that $[\alpha]^{\omega} \cap V$ is stationary in $[\alpha]^{\omega} \cap V[G]$, for every $G$ that is $P$-generic over $V$ (cf. [2], Definition 3.1). This weakens Shelah's concept of "properness." Recall that $P$ is called proper if for any $\alpha \geq \omega_{1}$ and any stationary $S \subset[\alpha]^{\omega} \cap V$ it is true that $S$ remains stationary in $[\alpha]^{\omega} \cap V[G]$, for every $G$ that is $P$-generic over $V$ (cf. [8]).

On the other hand, Woodin (unpublished) has shown that if the theory of $L(\mathbb{R})$ with real parameters from $V$ is frozen with respect to all set generic extensions whatsoever and symmetric submodels thereof, then in fact $A D^{L(\mathbb{R})}$ holds. (A version of this was rediscovered independently by Steel; cf. 11].) There is no denying the fact that a remarkable theorem of this sort provides one more bit of evidence for the naturalness (if not truth in $L(\mathbb{R})$ ) of $A D$, the Axiom of Determinacy.

Now in the light of [5] and [6] one obvious question is: can Woodin's result be strengthened by restricting the forcings to reasonable ones? This is non-trivial, as Woodin's proof uses the forcing for collapsing a singular cardinal to become countable, which is anything but reasonable. More specifically:

Question 1. Suppose that for every formula $\Phi(v)$, for every real $r \in \mathbb{R}^{V}$, for every $G$ that is $P$-generic, where $P \in V$ is reasonable, and for every symmetric submodel

Received by the editors April 24, 1998.

2000 Mathematics Subject Classification. Primary 03E55, 03E15; Secondary 03E35, 03E60.

Key words and phrases. Set theory, descriptive set theory, proper forcing, large cardinals.

The author would like to thank Itay Neeman, Philip Welch, and Sy Friedman for their interest and for their many hints and comments. John Steel even provided a crucial subclaim, and again I do say thanks for his intellectual support during my stay in Berkeley. I gratefully acknowledge financial support from the Deutsche Forschungsgemeinschaft (DFG).

(C)2000 American Mathematical Society 
$V^{\star}$ of $V[G]$,

$$
L\left(\mathbb{R}^{V}\right) \models \Phi(r) \quad \text { iff } \quad L\left(\mathbb{R}^{V^{\star}}\right) \models \Phi(r) .
$$

Does $A D^{L(\mathbb{R})}$ hold (in every set generic extension)?

We conjecture but cannot prove that the answer to this question is "yes." In this paper, we can only give partial evidence in favor of this conjecture. In fact, the argument given below can easily be transformed to show that under its assumption global $\boldsymbol{\Pi}_{1}^{1}$-determinacy holds.

If in particular $\omega_{1}$ is not to be collapsed, any attempt to answer this question in the affirmative seems to essentially have to use some coding techniques. As the coding is supposed to be set-sized, one cannot use Jensen coding as in [1] (although it is reasonable). A set-sized variant of it (as in [9], say) only works below $0^{\sharp}$ (or if $V$ is not closed under sharps, for that matter). In general, "coding into $K$ " techniques are called for, where $K$ is the core model, and this is what makes the problem really interesting (and difficult, once we get higher up in the large cardinal zoo).

So the above question naturally leads to the following:

Question 2. Suppose that $A D^{L(\mathbb{R})}$ does not hold. Let $X \subset \omega_{1}$. Can $X$ be coded into $L(\mathbb{R})$ by a set-sized reasonable forcing?

This would be a dual fact to the Anti-Coding Theorem of [5] and [6]. However, the transit via inner model theory for attacking this latter question is blocked at the time of writing by some pretty technical obstacles.

Let us now state the main results of the present paper.

Theorem 1.1. Suppose that there is no inner model with a strong cardinal. Let $X \subset \omega_{1}$. Then $X$ is $\boldsymbol{\Delta}_{3}^{1}$ (in the codes) in a generic extension by a set-sized reasonable forcing.

Theorem 1.2. Suppose that there is no inner model with a strong cardinal. Then there is a generic extension by a set-sized reasonable forcing with a $\boldsymbol{\Delta}_{3}^{1}$-well-ordering of its reals.

Theorem 1.3. Suppose that for every $\Sigma_{4}^{1}$-formula $\Phi(v)$, for every $G_{1}$ that is $P_{1}$ generic over $V$, where $P_{1} \in V$ is reasonable, for every real $r \in \mathbb{R}^{V\left[G_{1}\right]}$, and for every further $G_{2}$ that is $P_{2}$-generic over $V\left[G_{1}\right]$, where $P_{2} \in V\left[G_{1}\right]$ is reasonable,

$$
V\left[G_{1}\right] \models \Phi(r) \quad \text { iff } \quad V\left[G_{1}\right]\left[G_{2}\right] \models \Phi(r) .
$$

Then there is an inner model with a strong cardinal.

By the following result of Woodin (unpublished), 1.3 is best possible in the sense that one cannot derive more large-cardinal strength from its assumption. Let $\kappa$ be a strong cardinal, let $\lambda=2^{2^{\kappa}}$, and let $G$ be $\operatorname{Col}(\lambda, \omega)$-generic over $V$. Then any set generic extension $V[G]\left[H_{1}\right]$ of $V[G]$ is $\Sigma_{4}^{1}$-correct in any of its set generic extensions, $V[G]\left[H_{1}\right]\left[H_{2}\right]$. As 1.3 will be an immediate corollary to 1.2 , 1.2 itself is best possible in the sense that its anti-large cardinal assumption cannot be weakened.

Also, suppose $V$ to be the minimal inner model with one strong cardinal, say $V=$ " $\kappa$ is strong," and let $G$ be $\operatorname{Col}\left(\kappa^{++}, \omega\right)$-generic over $V$. By Woodin's result, $V[G]$ is $\Sigma_{4}^{1}$-correct in its set generic extensions. Writing $\omega_{1}=\omega_{1}^{V[G]}$, we have that $\mathcal{J}_{\omega_{1}}^{V}$ cannot be $\boldsymbol{\Sigma}_{3}^{1}$ in the codes: otherwise the $\boldsymbol{\Pi}_{4}^{1}$-statement

$$
\forall \text { countable } \xi \exists \text { countable } \alpha>\xi \rho_{\omega}\left(\mathcal{J}_{\alpha}^{V}\right) \leq \kappa^{++}
$$


would be true in $V[G]$, but could be made false by collapsing $\omega_{1}$, contradicting $\Sigma_{4}^{1}$-correctness. This implies that 1.1 is best possible, again in the sense that its anti-large-cardinal assumption cannot be weakened.

In subsequent work, [7], the present author has shown that neither in the statement of 1.1, nor of 1.2 nor of 1.3 can "remarkable" be replaced by "proper." (Cf. the discussion after the proof of Claim $3^{\prime}$.)

\section{Coding Below one Strong CARdinal}

Instead of directly aiming at proving [1.1 [1.2, and 1.3. we shall first present a reasonable generic extension of $V$ under the assumption that there is no inner model with a strong cardinal. This extension will be called $V_{4}$ below, and it will be

the case that in $V_{4}$ there is a real $a$ such that $H_{\omega_{2}}=J_{\omega_{2}}^{K(a)}$. (Here, $H_{\omega_{2}}$ is the set of all sets hereditarily smaller than $\aleph_{2}$, and $K(a)$ is the core model built over $a$, cf. the second next paragraph.) We shall then see that this construction in fact easily gives rise to proofs of 1.11 .2 and 1.3

So let us assume throughout this section that there is no inner model with a strong cardinal. Then $K$, the core model below a strong cardinal, exists (cf. [4]; we here have to assume just a little familiarity with $K$ ). Moreover, $K$ is also the core model in the sense of all set generic extensions. We shall code an initial segment of $V$ "into $K$." The heart of the matter will be the task of checking that a certain " $K$-reshaping" is $\omega$-distributive.

For this in turn it is convenient (although not necessary) at some point during the construction to switch from $K[A]$ to $K(A)$ for a set $A$ of ordinals, a distinction which should be explicitly explained. Fix $A$, a set of ordinals. Let $E$ code $K$ 's extender sequence, i.e., $K=L[E]$. Then by $K[A]$ we mean $L[E, A]$, i.e., the constructible universe built with the two additional predicates $v \in E$ and $v \in A$ at hand. Hence $K[A]$ is just the least inner model $W$ with $K \cup\{A\} \subset W$. We shall also write $K_{\kappa}[A]$ for $J_{\kappa}[E, A]$, for an ordinal $\kappa$. Notice that the presence of $A$ in $K[A]$ in general destroys the internal structure of $K$. On the other hand, by $K(A)$ we mean the core model built over $A$; i.e., starting from $T C(\{A\})$, the transitive closure of $\{A\}$, we run the recursive construction of $K$, with "strong $A$-mice" instead of "strong mice" (cf. [4] on the recursive definition of $K$; to give a reference for $K(A)$, cf. [10; p. 59). By our assumption that there is no inner model with a strong cardinal, $K(A)$ exists, and in contrast to $K[A]$ it has a fine structure and can be iterated "above $A . "$

With these things in mind, we may now commence with our construction.

To get things started, we use almost disjoint forcing in its simplest form. Fix $\delta$, a singular cardinal of uncountable cofinality and such that $\delta^{\aleph_{0}}=\delta$ (for example, let $\delta$ be a strong limit). We also may and shall assume that $\delta$ is a cutpoint of $K$; i.e., if $E_{\alpha} \neq \emptyset$ is an extender from $K$ 's extender sequence with $\alpha \geq \delta$, then in fact the citical point of $E_{\alpha}$ is $\geq \delta$, too. (Here we use that there is no inner model with a strong cardinal. If there were no such $\delta$ then using Fodor we would get a strong cardinal in $K$.)

By [4], we know that $\delta^{+K}=\delta^{+}$. We may also assume without loss of generality that $2^{\delta}=\delta^{+}$, because otherwise we may collapse $2^{\delta}$ onto $\delta^{+}$by a $\delta$-closed preliminary forcing. We may hence pick $A \subset \delta^{+}$with the property that $H_{\delta^{+}}=L_{\delta^{+}}[A]$. 
Now let $G_{1}$ be $\operatorname{Col}\left(\delta, \omega_{1}\right)$-generic over $V$. Notice that the forcing is $\omega$-closed. Set $V_{1}=V\left[G_{1}\right]$. We have that $\omega_{2}^{V_{1}}=\delta^{+}=\delta^{+K}$. Let $B \subset \omega_{1}$ code $G_{1}$ (in the sense that $\left.G_{1} \in L_{\omega_{2}^{V_{1}}}[B]\right)$.

Claim 1. In $V_{1}, H_{\omega_{2}}=L_{\omega_{2}}[A, B]$.

Proof. Easy, using the fact that $\operatorname{Col}\left(\delta, \omega_{1}\right)$ is $\delta^{+}$-c.c. We shall have to repeat the argument a couple of times, so we'll be more explicit next time. $\square$ (Claim 1)

In what follows we let $\omega_{2}$ denote $\omega_{2}^{V_{1}}$. It will also be the $\omega_{2}$ of all further extensions.

Now in $K$ we may pick $\left(A_{\xi}^{\prime}: \xi<\delta^{+}\right)$, a sequence of almost disjoint subsets of $\delta$. In $L_{\omega_{2}}[B]$ we may pick a bijective $g: \omega_{1} \rightarrow \delta$. Then, if we let $\alpha \in A_{\xi}$ iff $g(\alpha) \in A_{\xi}^{\prime}$ for $\alpha<\omega_{1}$ and $\xi<\delta^{+}$, we have that $\left(A_{\xi}: \xi<\delta^{+}\right)$is a sequence of almost disjoint subsets of $\omega_{1}$.

In $V_{1}$, we may pick $A_{1} \subset \omega_{2}$ with $H_{\omega_{2}}=L_{\omega_{2}}[A, B]=L_{\omega_{2}}\left[A_{1}\right]$ (for example, let $A_{1}=A \oplus B$, the "join" of $A$ and $B$ ). We let $P_{2}$ be the forcing for coding $A_{1}$ by a subset of $\omega_{1}$, using the almost disjoint sets $A_{\xi}$.

To be specific, $P_{2}$ consists of pairs $p=(l(p), r(p))$, where $l(p): \alpha \rightarrow 2$ for some $\alpha<\omega_{1}$ and $r(p)$ is a countable subset of $\omega_{2}$. We have $p=(l(p), r(p)) \leq_{P_{2}} q=$ $(l(q), r(q))$ iff $l(p) \supset l(q), r(p) \supset r(q)$, and for all $\xi \in r(q)$, if $\xi \in A_{1}$ then

$$
\{\beta \in \operatorname{dom}(l(p)) \backslash \operatorname{dom}(l(q)): l(p)(\beta)=1\} \cap A_{\xi}=\emptyset .
$$

By a $\Delta$-system argument, $P_{2}$ has the $\omega_{2}$-c.c. It is clearly $\omega$-closed, so no cardinals are collapsed. Moreover, if $G_{2}$ is $P_{2}$-generic over $V_{1}$, and if we set

$$
C^{\prime}=\bigcup_{p \in G_{2}}\{\beta \in \operatorname{dom}(l(p)): l(p)(\beta)=1\},
$$

then $C^{\prime} \subset \omega_{1}$, and we have that, for all $\xi<\omega_{2}$,

$$
\xi \in A_{1} \text { iff } \operatorname{Card}\left(C^{\prime} \cap A_{\xi}\right) \leq \aleph_{0}
$$

This means that $A_{1}$ is an element of any inner model containing $\left(A_{\xi}: \xi<\omega_{2}\right)$ and $C^{\prime}$. (Of course, much more holds.) Letting $C=B \oplus C^{\prime}$, an example of such a model is $K[C]$ in the sense explained above. Set $V_{2}=V_{1}\left[G_{2}\right]$. We then also have, by the same argument as for Claim 1,

Claim 2. In $V_{2}, H_{\omega_{2}}=K_{\omega_{2}}[C]$.

Proof. Let $X$ be a bounded subset of $\omega_{2}$ in $V_{2}$. As $P_{2}$ is $\omega_{2}$-c.c. and $P_{2} \in H_{\omega_{2}}$, $X$ has a name $\dot{X}$ in $H_{\omega_{2}}^{V_{1}}=L_{\omega_{2}}\left[A_{1}\right]$. That is, there is $\omega_{1}<\theta<\omega_{2}$ such that $\dot{X} \in L_{\theta}\left[A_{1} \cap \theta\right]$. Further, there is $\theta<\theta^{\prime}<\omega_{2}$ such that $\left(A_{\xi}: \xi<\theta\right) \in K_{\theta^{\prime}}[B]$, so that $A_{1} \cap \theta \in K_{\theta^{\prime}}\left[B \oplus C^{\prime}\right]=K_{\theta^{\prime}}[C]$. So $\dot{X} \in K_{\theta^{\prime}}[C]$, and hence $X \in K_{\omega_{2}}[C]$ by $C^{\prime} \in K_{\theta^{\prime}}[C]$.

We have shown that $H_{\omega_{2}} \subset K_{\omega_{2}}[C]$ in $V_{2}$. But $K_{\omega_{2}}[C] \subset H_{\omega_{2}}$ is trivial.

Now let $C_{1} \subset \omega_{1}$ be such that $C \in L_{\omega_{2}}\left[C_{1}\right]$ as well as $\mathcal{J}_{\delta}^{K} \in L_{\omega_{2}}\left[C_{1}\right]$ (for example, let $C_{1}=C \oplus$ a code for $\mathcal{J}_{\delta}^{K}$ ).

Our task is now to code "down to a real," i.e., we want to find a further $(\omega$ distributive) generic extension in which $H_{\omega_{2}} \subset K(a)$, where $a$ is a real. As we cannot expect $\omega_{1}$ to be a successor in $K$ (and as we certainly cannot force this 
to be the case using an $\omega_{1}$-preserving forcing), we have to use the slightly more advanced coding technique which first requires $C_{1}$ to become "reshaped."

However, there is no hope of showing that reshaping is reasonable if we work with the wrong definition of "being reshaped." As a matter of fact, the following one works.

Definition 2.1. Let $X \subset \omega_{1}$. We say that an $f$ is $X$-reshaping if $f: \alpha \rightarrow 2$ for some $\alpha \leq \omega_{1}$, and moreover, for all $\beta \leq \alpha$ with $\beta<\omega_{1}$,

$$
K\left(X \cap \beta, f\lceil\beta) \models \operatorname{Card}(\beta) \leq \aleph_{0} .\right.
$$

The reader will have noticed the round brackets in this definition. This indicates a convenient switch from $K[\cdots]$ to $K(\cdots)$ in our argument. It is also possible but virtually less elegant to keep going with the square brackets.

Now let $P_{3}$ be the forcing for adding a $C_{1}$-reshaping characteristic function on a subset of $\omega_{1}$, defined inside $V_{2}$. Formally, $p \in P_{3}$ iff $p$ is $C_{1}$-reshaping and $\operatorname{dom}(p)<\omega_{1}$. The order is by reverse inclusion, i.e., $q \leq_{P_{3}} p$ iff $q \supset p$. (In fact we may assume "w.l.o.g." that every $C_{1}$-reshaping $p$ has $\operatorname{dom}(p)<\omega_{1}$, because otherwise we could just fix a counterexample and go ahead with forcing with $P_{4}$.)

It is easy to see that for any $\alpha<\omega_{1}$, the set $D^{\alpha}=\left\{p \in P_{3}: \operatorname{dom}(p) \geq \alpha\right\}$ is open dense in $P_{3}$ (this property of $P_{3}$ is called its "extendability"). In fact, given $q \in P_{3}$ with $\operatorname{dom}(q)<\alpha$, we may pick any $p \leq q$ with $\operatorname{dom}(p)=\alpha+\omega$ such that $p\left\lceil[\operatorname{dom}(q), \operatorname{dom}(q)+\omega)\right.$ codes some bijective $f: \omega \rightarrow \alpha+\omega$; then $p \in D^{\alpha}$.

Claim 3. $P_{3}$ is $\omega$-distributive.

Proof. We first need the following observation, which is due to John Steel. The argument proving it also has to be repeated a couple of times.

Subclaim 1 (Steel). In $V_{2}, H_{\omega_{2}}=J_{\omega_{2}}^{K\left(C_{1}\right)}$.

Proof. Let $W=K^{K\left(C_{1}\right)}$, the core model built inside $K\left(C_{1}\right)$. In $V_{2}$, let $\theta$ be any regular cardinal, and let $\kappa$ be a singular cardinal with $c f(\kappa)>\theta$. By weak covering applied inside $K\left(C_{1}\right)$ (cf. 4) ), we have that $c f^{K\left(C_{1}\right)}\left(\kappa^{+W}\right) \geq \kappa$, which implies that $c f^{V_{2}}\left(\kappa^{+W}\right) \geq c f^{V_{2}}(\kappa)>\theta$. Hence, inside $V_{2}$, for any regular $\theta$ there is a stationary class of cardinals $\kappa$ with $c f\left(\kappa^{+W}\right)>\theta$.

But this implies that inside $V_{2}, W$ is a universal weasel in the sense that the coiteration of $W$ with any (set-sized) mouse terminates after $<O R$ many steps and the mouse-side is always simple.

Moreover, as $\mathcal{J}_{\delta}^{K} \in K\left(C_{1}\right)$, an easy absoluteness argument using the recursive definition of $K$ (cf. [4] or [10], p. 85 f.) yields that $\mathcal{J}_{\delta}^{K} \triangleleft W$, i.e., $\mathcal{J}_{\delta}^{K}$ is an initial segment of $W$.

By [4, there is a normal iteration of $K$ giving $W$ (cf. also [10], Theorem 8.13). Let $\pi: K \rightarrow W$ be the iteration map. Because $\delta$ is a cutpoint in $K$ and $\mathcal{J}_{\delta}^{K} \triangleleft W$, we must have $\pi\left\lceil\delta=i d\right.$. But as $\mathcal{J}_{\delta+K}^{K}$ is an initial segment of every simple iterate of $K$ above $\delta$, this readily implies that $\mathcal{J}_{\delta^{+} W}^{W}=\mathcal{J}_{\delta^{+} K}^{K}$.

Hence $J_{\omega_{2}}^{K} \in W \subset K\left(C_{1}\right)$, and in fact $J_{\omega_{2}}^{K} \subset J_{\omega_{2}}^{K\left(C_{1}\right)}$ by acceptability. This easily gives the claim.

We remark in passing that Subclaim 1 would not have to hold if $\delta$ had not been chosen as a cutpoint of $K$.

We now fix a condition $p \in P_{3}$, and open dense sets $D_{i}, i<\omega$. We have to find $q \leq_{P_{3}} p$ with $q \in D_{i}$ for every $i<\omega$. We are working in $V_{2}$. Let us consider the 
model

$$
\mathfrak{M}=\left(H_{\omega_{2}} ; \in, C_{1}, E^{K\left(C_{1}\right)} \uparrow \omega_{2},\left(D_{i}: i<\omega\right), p\right),
$$

where $E^{K\left(C_{1}\right)}$ is the extender sequence from $K\left(C_{1}\right)$. Let $X_{0}$ be the universe of the least $\Sigma_{1}$ submodel of $\mathfrak{M}$, and for $n>0$ let $X_{n}$ be the universe of the least $\Sigma_{1}$ submodel of $\mathfrak{M}$ containing $X_{n-1} \cup\left\{X_{n-1}\right\}$. Here, "least" refers to some fixed $\Sigma_{1}$ Skolem function for $\mathfrak{M}$ which is definable over $\mathfrak{M}$. Let $X_{\omega}=\bigcup_{n<\omega} X_{n}$. Of course, every $X_{n}, n \leq \omega$, is countable. For $n \leq \omega$, let

$$
\pi_{n}: \mathfrak{N}_{n}=\left(\bar{H}_{n} ; \in, C_{1} \cap \kappa_{n}, \bar{E}_{n},\left(\bar{D}_{i, n}: i<\omega\right), p\right) \rightarrow_{\Sigma_{1}}\left(X_{n} ; \ldots\right) \prec \mathfrak{M}
$$

be the inverse of the Mostowski collapse of $X_{n}$, where $\kappa_{n}=c . p .\left(\pi_{n}\right)$ is the critical point of $\pi_{n}$. (Of course, $\pi_{n}\left(\kappa_{n}\right)=\omega_{1}$.) Let us write $\pi=\pi_{\omega}, \mathfrak{N}=\mathfrak{N}_{\omega}$, etc. in what follows. It is crucial to notice that $\left(\kappa_{n}: n<\omega\right)$ is definable over $\mathfrak{N}$, as $\pi^{-1} " X_{n}$ is the least $\Sigma_{1}$ submodel of $\mathfrak{N}$ (containing $\pi^{-1} " X_{n-1} \cup\left\{\pi^{-1} " X_{n-1}\right\}$ for $n>0$ ).

Subclaim 2. $\mathfrak{N} \in K\left(C_{1} \cap \kappa\right)$.

Proof. $\bar{H}$ is the universe of a $\left(C_{1} \cap \kappa\right)$-premouse, call it $N$, with extender sequence $\bar{E}$. Obviously, it suffices to prove that $N \in K\left(C_{1} \cap \kappa\right) . N$ is iterable, as witnessed by $\pi: N \rightarrow \Sigma_{1} \mathcal{J}_{\omega_{2}}^{K\left(C_{1}\right)}$. We have that $\rho_{1}(N) \leq \kappa$, as $N$ is the $\Sigma_{1}$ hull of $\kappa \cup$ $\left\{\left(\bar{D}_{i}: i<\omega\right), p\right\}$ taken inside $N$. Moreover, $N$ is sound: as it does not have any total extenders on its sequence, it can't be a proper iterate of its core.

We coiterate $N$ with $K\left(C_{1} \cap \kappa\right)$, getting comparable $N^{*}$ and $K^{*}$. Because $\rho_{1}(N) \leq \kappa$, no non-trivial iterate of $N$ can be sound. This implies that, by the universality of $K\left(C_{1} \cap \kappa\right), N$ cannot be moved at all in the comparison, i.e., $N^{*}=N$.

Now suppose that $K\left(C_{1} \cap \kappa\right)$ were to be moved, i.e., $K^{*} \neq K\left(C_{1} \cap \kappa\right)$, Let $\nu$ be the index of the first extender of the iteration from $K\left(C_{1} \cap \kappa\right)$ to $K^{*}$. We have that $\nu$ is a cardinal in $K^{*}$. But then $\nu>N \cap O R$, as $\rho_{1}(N) \leq \kappa$ and $N \unlhd K^{*}$. This means that there was no need to iterate $K\left(C_{1} \cap \kappa\right)$ at all, so that in fact $N \triangleleft K\left(C_{1} \cap \kappa\right)$. In particular, $N \in K\left(C_{1} \cap \kappa\right)$.

(Subclaim 2)

By the remark right before the statement of Subclaim 2 we thus have that

$$
K\left(C_{1} \cap \kappa\right) \models \operatorname{Card}(\kappa) \leq \aleph_{0} .
$$

We are now going to construct a sequence $\left(p_{i}: i<\omega\right)$ of conditions below $p$ such that $p_{i+1} \leq_{P_{3}} p_{i}$ and $p_{i+1} \in D_{i}$ for all $i<\omega$. We also want to maintain inductively that $p_{i} \in \bar{H}_{i}$ for all $i<\omega$. To commence, let $p_{0}=p$. (Notice that $p_{0} \in \bar{H}_{0}$.) Now suppose that $p_{i}$ is given, $p_{i} \in \bar{H}_{i}$, some $i<\omega$. Then $\operatorname{dom}\left(p_{i}\right)<\kappa_{i}$, and $p_{i} \in \bar{H}_{i+1}$, too. Applying the extendability of $\pi_{i+1}^{-1}\left(P_{3}\right)$ and the density of $\bar{D}_{i, i+1}$ inside $\mathfrak{N}_{i+1}$, it is easy to find $p_{i+1} \leq_{P_{3}} p_{i}$ such that $p_{i+1} \in \bar{H}_{i+1}, p_{i+1} \in \bar{D}_{i, i+1} \subset D_{i, i+1}$, and $\operatorname{dom}\left(p_{i+1}\right) \geq \kappa_{i}$.

Now set $q=\bigcup_{i<\omega} p_{i}$. We have arranged that $\operatorname{dom}(q)=\bigcup_{i<\omega} \kappa_{i}=\kappa$. But this implies that $q$ is $C_{1}$-reshaping, because $K\left(C_{1} \cap \kappa, q\right) \models \operatorname{Card}(\kappa) \leq \aleph_{0}$. (We even have that $K\left(C_{1} \cap \kappa\right)=\operatorname{Card}(\kappa) \leq \aleph_{0}$.) Thus $q \in P_{3}$, and $q$ is as desired.

$\square$ (Claim 3)

As a matter of fact, a somewhat more involved variation of the previous argument shows that $P_{3}$ is stationary preserving. (Recall that a notion of forcing $P$ is called stationary preserving if every stationary $S \subset \omega_{1}$ remains stationary in any generic extension obtained from forcing with $P$.)

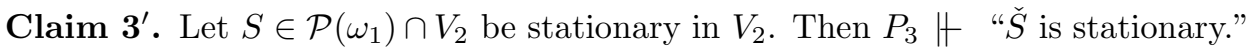


Proof. Let $r \in P_{3}$ be such that $r \nVdash$ " $\dot{C}$ is a club subset of $\omega_{1}$." W.l.o.g., $\dot{C} \in H_{\omega_{2}}$. Let $p \leq_{P_{3}} r$, and consider the model

$$
\mathfrak{M}=\left(H_{\omega_{2}} ; \in, C_{1}, E^{K\left(C_{1}\right)} \uparrow \omega_{2}, \dot{C}, p\right) .
$$

Let $C^{\star} \subset \omega_{1}$ be the set of all $\kappa<\omega_{1}$ such that if $X$ is the universe of the least $\Sigma_{1}$ submodel of $\mathfrak{M}$ containing $\kappa$, then $\kappa=X \cap \omega_{1}$. Obviously, $C^{\star}$ is club in $\omega_{1}$, so that we may pick some $\kappa \in S \cap C^{\star}$. We aim to construct $q \leq_{P_{3}} p$ with $q \| \check{\kappa} \in \dot{C}$.

Let $X$ witness $\kappa \in C^{\star}$, and let

$$
\pi: \mathfrak{N}=\left(\bar{H} ; \in, C_{1} \cap \kappa, \bar{E}, \bar{C}, p\right) \rightarrow \Sigma_{1}(X ; \ldots) \prec \mathfrak{M}
$$

be the inverse of the Mostowski collapse of $X$. Unfortunately, we won't have in general that $K\left(C_{1} \cap \kappa\right) \models \operatorname{Card}(\kappa) \leq \aleph_{0}$ as in the proof of Claim 3. However, if we let $N$ be defined exactly as there, we shall again have that $N$ is sound, and $\rho_{1}(N) \leq \kappa$; then $N \triangleleft K\left(C_{1} \cap \kappa\right)$, and in fact $N \triangleleft \mathcal{J}_{\kappa^{+K\left(C_{1} \cap \kappa\right)}}^{K\left(C_{1} \cap \kappa\right)}$. This will enable us to use an argument from [9] to build $q$.

We may assume w.l.o.g. that $\kappa=\omega_{1}^{K\left(C_{1} \cap \kappa\right)}$, as otherwise $\kappa$ is countable in $K\left(C_{1} \cap \kappa\right)$ and the argument collapses to the one for Claim 3. As $N$ has size $\kappa$ in $K\left(C_{1} \cap \kappa\right)$, we may pick a club $E \subset \kappa$ in $K\left(C_{1} \cap \kappa\right)$ which grows faster than all clubs in $N$, i.e., whenever $\bar{E} \subset \kappa$ is a club in $N$ then $E \backslash \bar{E}$ is bounded in $\kappa$. Let $\left(\lambda_{i}: i<\omega\right)$ be a sequence of ordinals below $\kappa$ which is cofinal in $\kappa$. Let $D_{i}=\left\{s: \operatorname{dom}(s) \geq \lambda_{i} \wedge \exists \alpha \geq \lambda_{i} s \nVdash \check{\alpha} \in \dot{C}\right\}$, for $i<\omega$. Then $\left(D_{i}: i<\omega\right) \subset \operatorname{ran}(\pi)$ is a sequence of open dense subsets of $P_{3}$.

We are now going to construct a sequence $\left(p_{i}: i<\omega\right)$ of conditions below $p$ such that $p_{i+1} \leq_{P_{3}} p_{i}$ and $p_{i+1} \in D_{i}$. We also want to maintain inductively that $p_{i+1} \in \bar{H}$. (Notice that $p \in \bar{H}$ to begin with.)

To commence, let $p_{0}=p$. Now suppose that $p_{i}$ is given, $p_{i} \in N$. Set $\alpha=$ $\operatorname{dom}\left(p_{i}\right)<\kappa$. Work inside $N$ for a minute. For all $\beta$ such that $\alpha \leq \beta<\kappa$ we may pick some $p^{\beta} \leq_{P_{3}} p_{i}$ such that $p^{\beta} \in \pi^{-1}\left(D_{i}\right), \operatorname{dom}\left(p^{\beta}\right)>\beta$, and for all limit ordinals $\lambda, \alpha \leq \lambda \leq \beta, p^{\beta}(\lambda)=1$ iff $\lambda=\beta$. Then there is $\bar{E}$ club in $\kappa$ such that for any $\eta \in \bar{E}, \beta<\eta \Rightarrow \operatorname{dom}\left(p^{\beta}\right)<\eta$.

Now back in $K\left(C_{1} \cap \kappa\right)$, we may pick $\beta \in E$ such that $E \backslash \bar{E} \subset \beta$. Set $p_{i+1}=p^{\beta}$, and for future reference let $\beta=\beta_{i+1}$. Of course $p_{i+1} \in D_{i} \cap \bar{H}$. We also have that $\operatorname{dom}\left(p_{i+1}\right)<\min \{\epsilon \in E: \epsilon>\beta\}$, so that for all limit ordinals $\lambda \in E \cap$ $\left(\operatorname{dom}\left(p_{i+1}\right) \backslash \operatorname{dom}\left(p_{i}\right)\right)$ we have that $p_{i+1}(\lambda)=1$ iff $\lambda=\beta_{i+1}$.

Now set $q=\bigcup_{i<\omega} p_{i}$. We have arranged that $\operatorname{dom}(q)=\bigcup_{i<\omega} \lambda_{i}=\kappa$. The only problem left to verify is that $q$ is a condition, because if so then clearly $q \Vdash \check{\kappa} \in \dot{C}$; i.e., we want to see that

$$
K\left(C_{1} \cap \kappa, q\right) \models \operatorname{Card}(\kappa) \leq \aleph_{0} .
$$

But by the construction of the $p_{i}$ 's we have that

$$
\begin{gathered}
\{\lambda \in E \cap(\operatorname{dom}(q) \backslash \operatorname{dom}(p)): \lambda \text { is a limit ordinal and } q(\lambda)=1\} \\
=\left\{\beta_{i+1}: i<\omega\right\},
\end{gathered}
$$

which is a cofinal subset of $E$. It hence suffices to verify that $E$ is an element of $K\left(C_{1} \cap \kappa, q\right)$, because then $\left\{\beta_{i+1}: i<\omega\right\} \in K\left(C_{1} \cap \kappa, q\right)$ witnesses that $\operatorname{Card}(\kappa) \leq \aleph_{0}$.

But $E \in K\left(C_{1} \cap \kappa, q\right)$ is shown by the argument for Subclaim 1: we build $W=K\left(C_{1} \cap \kappa\right)^{K\left(C_{1} \cap \kappa, q\right)}$, observe its universality in $V_{2}$, and deduce that $E \in$ $J_{\omega_{2}}^{K\left(C_{1} \cap \kappa\right)}=J_{\omega_{2}}^{W} \subset K\left(C_{1} \cap \kappa, q\right)$.

$\square\left(\right.$ Claim $\left.3^{\prime}\right)$ 
On the other hand, it is shown in [7] that $P_{3}$ is not always proper. In fact, if $V$ is obtained from $L$ by Levy collapsing a remarkable cardinal in $L$ (in particular, $0^{\sharp}$ does not exist and $\left.K=L\right)$, then no $L(\mathbb{R})$ of any proper set forcing extension whatsoever can contain an $\omega_{1}$ sequence of pairwise distinct reals.

Now let $G_{3}$ be $P_{3}$-generic over $V_{2}$, and set $V_{3}=V_{2}\left[G_{3}\right]$. By the extendability of $P_{3}$ we have that $\bigcup G_{3}$ is a $C_{1}$-reshaping function with domain $\omega_{1}$. Let $C^{\prime \prime}$ be that subset of $\omega_{1}$ having $\bigcup G_{3}$ as its characteristic function, and let $D$ code $C_{1} \oplus C^{\prime \prime}$. Again we get:

Claim 4. In $V_{3}, H_{\omega_{2}}=J_{\omega_{2}}^{K(D)}$.

Proof. The only new point here is that we have to check that $J_{\omega_{2}}^{K\left(C_{1}\right)} \subset J_{\omega_{2}}^{K(D)}$. But here we can argue exactly as for Subclaim 1 above, by building $K\left(C_{1}\right)^{K(D)}$, observing its universality, and deducing that $J_{\omega_{2}}^{K\left(C_{1}\right)} \subset K(D) . \quad \square$ (Claim 4)

We may now finally code down to a real by using almost disjoint forcing once more. By the fact that $D$ is " $K$-reshaped," there is a (unique) sequence $\left(a_{\beta}: \beta<\right.$ $\left.\omega_{1}\right)$ of subsets of $\omega$ such that for each $\beta<\omega_{1}, a_{\beta}$ is the $K(D \cap \beta)$-least subset of $\omega$ that is almost disjoint from any $a_{\bar{\beta}}$ for $\bar{\beta}<\beta$.

We then let $P_{4}$ consist of all pairs $p=(l(p), r(p))$, where $l(p): n \rightarrow 2$ for some $n<\omega$ and $r(p)$ is a finite subset of $\omega_{1}$. We let $p=(l(p), r(p)) \leq_{P_{4}} q=(l(q), r(q))$ iff $l(p) \supset l(q), r(p) \supset r(q)$, and for all $\beta \in r(q)$, if $\beta \in D$ then

$$
\{\gamma \in \operatorname{dom}(r(p)) \backslash \operatorname{dom}(r(q)): r(p)(\gamma)=1\} \cap a_{\beta}=\emptyset .
$$

By another $\Delta$-system argument, $P_{4}$ has the c.c.c. Moreover, if $G_{4}$ is $P_{4}$-generic over $V_{3}$, and if we set

$$
a=\bigcup_{p \in G_{4}}\{\gamma \in \operatorname{dom}(l(p)): l(p)(\gamma)=1\}
$$

then we have that for $\gamma<\omega_{1}$,

$$
\gamma \in D \text { iff } \operatorname{Card}\left(a \cap a_{\gamma}\right)<\aleph_{0}
$$

We finally get

Claim 5. In $V_{4}, H_{\omega_{2}}=J_{\omega_{2}}^{K(a)}$.

Proof. In order to show that $J_{\omega_{2}}^{K(D)} \subset K(a)$ we first have to verify $D \in K(a)$. This is easily seen to follow (combined with the uniform definability of the $a_{\beta}$ 's) from the fact that if $D \cap \gamma \in K(a)$ then $J_{\omega_{2}}^{K(D \cap \gamma)} \subset K(a)$ (which in turn is true by the argument for Subclaim 1).

But then one more Subclaim 1 type argument proves $J_{\omega_{2}}^{K(D)} \subset K(a)$.

At last, we observe that the 4-step iteration yielding from $V$ to $V_{4}$ is reasonable. Let $\alpha$ be any uncountable ordinal. Because $P_{1}, P_{2}$, and $P_{3}$ are all $\omega$-distributive, we have that $[\alpha]^{\omega} \cap V=[\alpha]^{\omega} \cap V_{3}$. But $P_{4}$ is c.c.c., hence proper, which implies that $[\alpha]^{\omega} \cap V$ is stationary in $[\alpha]^{\omega} \cap V_{4}$.

Actually, we also get that every $S \in \mathcal{P}\left(\omega_{1}\right) \cap V$ which is stationary in $V$ remains stationary in $V_{4}$. Clearly, any such $S$ is still stationary in $V_{2}$, as $P_{1}$ and $P_{2}$ are both $\omega$-closed, hence proper. But then $S$ is stationary in $V_{3}$ by Claim $3^{\prime}$, and so in the end $S$ is still stationary in $V_{4}$ by the properness of $P_{4}$. In other words, the 4-step iteration from $V$ to $V_{4}$ is also stationary preserving. 


\section{Getting the theorems}

We may now easily derive $1.1,1.2$ and 1.3 from the work done in the previous section.

Proof of 1.1. Let us assume that there is no inner model with a strong cardinal. Fix $X \subset \omega_{1}$. Running the construction of the last section, we may certainly assume w.l.o.g. that for all $\xi<\omega_{1}, \xi \in X$ iff $2 \xi \in D$. But then we have that in $V_{4}, \xi \in X$ iff

$$
\begin{gathered}
\exists\left(a_{\gamma}: \gamma \leq 2 \xi\right) \exists d \subset 2 \xi+1\left[\forall \gamma \leq 2 \xi\left(a_{\gamma} \text { is the } K(d \cap \gamma)\right. \text {-least }\right. \\
\text { subset of } \omega \text { a.d. from all } a_{\bar{\gamma}}, \bar{\gamma}<\gamma, \\
\text { and } \left.\left.\gamma \in d \leftrightarrow \operatorname{Card}\left(a \cap a_{\gamma}\right)<\aleph_{0}\right) \text { and } 2 \xi \in d\right] .
\end{gathered}
$$

But $a_{\gamma}$ is the $K(d \cap \gamma)$-least subset of $\omega$ a.d. from all $a_{\bar{\gamma}}, \bar{\gamma}<\gamma$, iff $a_{\gamma}$ is the $\mathcal{M}$-least subset of $\omega$ a.d. from all $a_{\bar{\gamma}}, \bar{\gamma}<\gamma$, for all $(d \cap \gamma)$-mice $\mathcal{M}$ with $\left\{a_{\bar{\gamma}}: \bar{\gamma} \leq \gamma\right\} \subset \mathcal{M}$. We may hence rewrite the displayed formula as

$$
\begin{aligned}
& \exists\left(a_{\gamma}: \gamma \leq 2 \xi\right) \exists d \subset 2 \xi+1 \exists\left(\mathcal{M}_{\gamma}: \gamma \leq 2 \xi\right)\left[\forall \gamma \leq 2 \xi\left(\mathcal{M}_{\gamma}\right. \text { is a }\right. \\
& \quad(d \cap \gamma) \text {-mouse with }\left\{a_{\bar{\gamma}}: \bar{\gamma} \leq \gamma\right\} \subset \mathcal{M}_{\gamma}, \\
& a_{\gamma} \text { is the } \mathcal{M}_{\gamma} \text {-least subset of } \omega \text { a.d. from all } a_{\bar{\gamma}}, \bar{\gamma}<\gamma, \\
& \text { and } \left.\left.\gamma \in d \leftrightarrow \operatorname{Card}\left(a \cap a_{\gamma}\right)<\aleph_{0}\right) \text { and } 2 \xi \in d\right] .
\end{aligned}
$$

As "mousehood" is $\Pi_{2}^{1}$, this latter displayed formula is now easily be seen to be $\Sigma_{3}^{1}(a)$. Hence $X$ turns out to be $\Sigma_{3}^{1}(a)$ in the codes, inside $V_{4}$.

But because $\left(a_{\gamma}: \gamma<\omega_{1}\right)$ is uniquely determined given $a$, it is easy that $X$ is now $\Pi_{3}^{1}(a)$ in the codes as well, inside $V_{4}$.

There is also a uniform version of 1.1: if there is no inner model with a strong cardinal, then there is a generic extension by a set-sized reasonable forcing in which every $X \subset \omega_{1}$ will be $\boldsymbol{\Delta}_{3}^{1}$ in the codes. To prove this, instead of forcing with what we called $P_{4}$ over $V_{3}$, we force Martin's Axiom instead.

Proof of 1.2. Assuming that there is no inner model with a strong cardinal and building $V_{4}$ as in the last section, we have by Claim 5 that $H_{\omega_{2}}=J_{\omega_{2}}^{K(a)}$ in $V_{4}$. Inside $V_{4}$, we may thus define a well-ordering of the reals by $x<y$ iff

$$
\exists \mathcal{M}\left[\mathcal{M} \text { is an } a \text {-mouse and } x<_{\mathcal{M}} y\right] .
$$

(Here, $<_{\mathcal{M}}$ denotes the order of constructibility of $\mathcal{M}$.) Again using the fact that "mousehood" is $\Pi_{2}^{1}$, this gives a $\Sigma_{3}^{1}(a)$-wellordering of $\mathbb{R}$. But clearly $x<y$ can now also written in a $\Pi_{3}^{1}(a)$-fashion.

Proof of 1.3. Let us first suppose that there is a $\boldsymbol{\Pi}_{3}^{1}$-well-ordering of $\mathbb{R}$. This fact can be expressed by a $\boldsymbol{\Pi}_{4}^{1}$-statement $\Phi$ that is true in $V$. By adding $\omega_{1}$ many Cohen reals with finite support we get a (c.c.c., hence proper, hence) reasonable extension $V[G]$ of $V$ in which there is no projective well-ordering of the reals, so that $\Phi$ fails.

Thus if the assumption of 1.3 holds, then there can be no $\Pi_{3}^{1}$-well-ordering of the reals in $V$. Now let us suppose that there is no inner model with a strong cardinal.

Then by 1.2 there is a reasonable extension $V[G]$ of $V$ with a $\Pi_{3^{-}}^{1}$ (in fact $\boldsymbol{\Delta}_{3^{-}}^{1}$ ) well-ordering of its reals. As above, this fact can be expressed by a $\boldsymbol{\Pi}_{4}^{1}$-statement $\Phi$ that is true in $V[G]$. But again by adding $\omega_{1}$ many Cohen reals we get a reasonable extension $V[G][H]$ of $V[G]$ in which there is no projective well-ordering of the reals, so that $\Phi$ fails. Contradiction! 
We have shown that there is an inner model with a strong cardinal. $\quad \square$ (1.3)

By the remark at the end of the previous section, one can replace "reasonable" by "stationary preserving" in the statements of $1.1,1.2$ and 1.3 .

\section{FURTHER REMARKS}

In this last section we want to point out a couple of things which are of related interest. The first one is an immediate corollary to 1.2, combined with an old argument of Harrington (cf. 3], pp. 575 ff.).

Fact 1. Suppose that there is no inner model with a strong cardinal. Let $\delta$ be any ordinal. Then there is a generic extension by a set-sized reasonable forcing in which $\boldsymbol{\delta}_{4}^{1}>\max \left\{\delta, \aleph_{3}\right\}$.

If $V$ is not closed under $\sharp$ 's, then a slight variation of the Harrington argument actually shows that we can make $\boldsymbol{\delta}_{3}^{1}$ as large as prescribed by a set-sized reasonable forcing. On the other hand, if $\mathbb{R}$ is closed under $\sharp$ 's (i.e., if $\boldsymbol{\Pi}_{1}^{1}$-determinacy holds), then $\boldsymbol{\delta}_{3}^{1} \leq \aleph_{3}$.

Proof of Fact 1 (sketch). Going first to a reasonable extension provided by 1.2, we then run the Harrington construction as in [3], pp. 575 ff., say. As the two further forcings are c.c.c., hence proper, the final extension is a reasonable one. $\square$ (Fact 1 , sketch)

Finally, we want to point out a "converse" to 1.2 which was noted in 13 , Theorem $6(b)$.

Fact 2. Suppose that there is no inner model with a strong cardinal, and $\mathbb{R}$ is closed under $\sharp$ 's (i.e., $\Pi_{1}^{1}$-determinacy holds). Let $a \in \mathbb{R}$. If there is a $\Sigma_{3}^{1}(a)$-well-ordering of $\mathbb{R}$, then in fact $\mathbb{R}=\mathbb{R} \cap K(a)$.

This is false if $\mathbb{R}$ is not closed under $\sharp$ 's. On the other hand, we can weaken the anti-large-cardinal assumption to "there is no inner model with a Woodin cardinal" if in addition we are willing to assume the existence of two measurable cardinals (in $V$ ) (this follows from Steel's $\Sigma_{3}^{1}$-correctness theorem, cf. [10]).

Proof of Fact 2 (sketch). Under the hypotheses of the theorem, by [12] there is a tree $T_{2} \in K(a)$ projecting to the universal $\Pi_{2}^{1}$-set of reals. (This tree gives $\Sigma_{3}^{1}$ correctness of $K(a)$ in $V$.) But we can now run the Mansfield proof that if there is a $\Sigma_{2}^{1}(b)$-well-ordering of the reals, then in fact $\mathbb{R} \subset L[b]$, for $b \in \mathbb{R}$ (cf. [3] , Theorem 100 (c), p. 534), but with the Shoenfield tree replaced by $T_{2}$. This gives the desired result.

$\square$ (Fact 2, sketch)

As a by-product of this proof we get that if $A$ is a $\Sigma_{3}^{1}(a)$-set of reals, $a \in \mathbb{R}$, and $A \backslash K(a) \neq \emptyset$, then $A$ in fact has a perfect subset. Hence if $\omega_{1}^{K(a)}$ is countable for every $a \in \mathbb{R}$, then every $\boldsymbol{\Sigma}_{3}^{1}$-set of reals has the perfect subset property. (This appears in [14, p. 286.)

\section{REFERENCES}

[1] Beller, A., Jensen, R., and Welch, Ph., Coding the universe, Cambridge 1982. MR 84b:03002

[2] Foreman, M., and Magidor, M., Large cardinals and definable counterexamples to the continuum hypothesis, Ann. Pure Appl. Logic 76 (1995), pp. 47 - 97. MR 96k:03124

[3] Jech, T., Set theory, San Diego 1978. MR 80a:03062 
[4] Jensen, R., The core model for non-overlapping extender sequences, handwritten notes.

[5] Neeman, I., and Zapletal, J., Proper forcing and $L(\mathbb{R})$, preprint.

[6] - Proper forcing and absoluteness in $L(\mathbb{R})$, Comment. Math. Univ. Carolinae 39 (1998), pp. 281 - 301. CMP 99:03

[7] Schindler, R.-D., Proper forcing and remarkable cardinals, to appear.

[8] Shelah, S., Proper forcing, Springer-Verlag 1982. MR 84h:03042

[9] _ and Stanley, L., Coding and reshaping when there are no sharps, in: "Set theory of the continuum," Judah, H., et al. (eds.), Springer Verlag 1992, pp. 407 - 416. MR 94m:03083

[10] Steel, J., The core model iterability problem, Springer Verlag 1996. MR 99k:03043

[11] _ Core models with more Woodin cardinals, preprint.

[12] of Mathematics 104 (1998), pp. 157 - 190. MR 99d:03044

[13] Welch, P., Doing without determinacy, in: "Proc. Logic Colloq. 86, Drake and Truss (eds.), North Holland 1988, pp. 333 - 342. MR 88k:03104

[14] Some descriptive set theory and core models, Ann. Pure Appl. Logic 39 (1988), pp. 273 - 290. MR 90d:03095

Department of Mathematics, University of California, Berkeley, California 94720 Current address: Institut für formale Logik, Universität Wien, 1090 Wien, Austria

E-mail address: rds@logic.univie.ac.at 